\title{
CDISC SDTM Microscopic Findings Test Detail Terminology
}

National Cancer Institute

\section{Source}

National Cancer Institute. CDISC SDTM Microscopic Findings Test Detail Terminology.

NCl Thesaurus. Code C125922.

Terminology associated with the microscopic findings test detail codelist of the Clinical

Data Interchange Standards Consortium (CDISC) Study Data Tabulation Model (SDT M). 